\title{
Equivalent circuit simulation of HPEM-induced transient responses at nonlinear loads
}

\author{
Miroslav Kotzev ${ }^{1}$, Xiaotang Bi ${ }^{1}$, Matthias Kreitlow ${ }^{2}$, and Frank Gronwald ${ }^{1}$ \\ ${ }^{1}$ Institute for Reliability of Technical Systems and Electrical Measurement, University of Siegen, Hölderlinstr. 3, \\ 57076 Siegen, Germany \\ ${ }^{2}$ Bundeswehr Research Institute for Protective Technologies and NBC Protection, Humboldtstr. 100, \\ 29663 Munster, Germany \\ Correspondence to: Frank Gronwald (frank.gronwald@uni-siegen.de)
}

Received: 15 January 2017 - Accepted: 25 April 2017 - Published: 21 September 2017

\begin{abstract}
In this paper the equivalent circuit modeling of a nonlinearly loaded loop antenna and its transient responses to HPEM field excitations are investigated. For the circuit modeling the general strategy to characterize the nonlinearly loaded antenna by a linear and a nonlinear circuit part is pursued. The linear circuit part can be determined by standard methods of antenna theory and numerical field computation. The modeling of the nonlinear circuit part requires realistic circuit models of the nonlinear loads that are given by Schottky diodes. Combining both parts, appropriate circuit models are obtained and analyzed by means of a standard SPICE circuit simulator. It is the main result that in this way fullwave simulation results can be reproduced. Furthermore it is clearly seen that the equivalent circuit modeling offers considerable advantages with respect to computation speed and also leads to improved physical insights regarding the coupling between HPEM field excitation and nonlinearly loaded loop antenna.
\end{abstract}

\section{Introduction}

The effects of high power electromagnetic (HPEM) sources on electric and electronic systems have been the subject of many studies during the last decades. Usually, the corresponding physical models focus on the formulation and solution of electromagnetic boundary value problems. Most of these models employ linear constitutive equations such that the usual methods of classical electrodynamics can be used for their solution (Lee, 1995; Tesche et al., 1997). The presence of electronic elements, however, implies that nonlinear effects may also need to be taken into account. In a recent study, a measurement setup has been proposed that allows to produce nonlinear effects that are due to transient HPEM excitation of a canonical system, compare Fig. 1. Additionally, it was possible to correlate the corresponding measurement results to numerical full wave simulations in time domain (Kotzev et al., 2016). A corresponding simulation model consisting of a nonlinearly loaded loop antenna placed inside a metallic resonator is depicted in Fig. 2. Similar to the measurement setup, in the model the resonator is excited by a transient plane wave which couples via a horizontal slot into the resonator.

It is the aim of this paper to supplement the obtained results by SPICE circuit models and simulation. For this purpose, appropriate equivalent circuit models are required. Such models can be derived based on classical techniques such as the singularity expansion method (Michalski and Pearson, 1984) or by the characteristic modes representation of antenna impedances and electromagnetic fields (Adams and Bernhard, 2013). Also, effective lumped-element equivalent circuits of microwave circuits can be obtained with the help of the transmission line matrix (TLM) method as shown in Russer et al. (1994) and Felsen et al. (2009). In the present context the determination of equivalent circuit models mainly follows the methods for the extraction of broadband equivalent circuits of loop and dipole antennas as suggested by Streable and Pearson (1981), Long et al. (2000), and Rambabu et al. (1999).

The paper is organized as follows: in the next Sect. 2 an equivalent circuit model of the considered loop antenna in free space is derived. In Sect. 3 the equivalent circuit model 


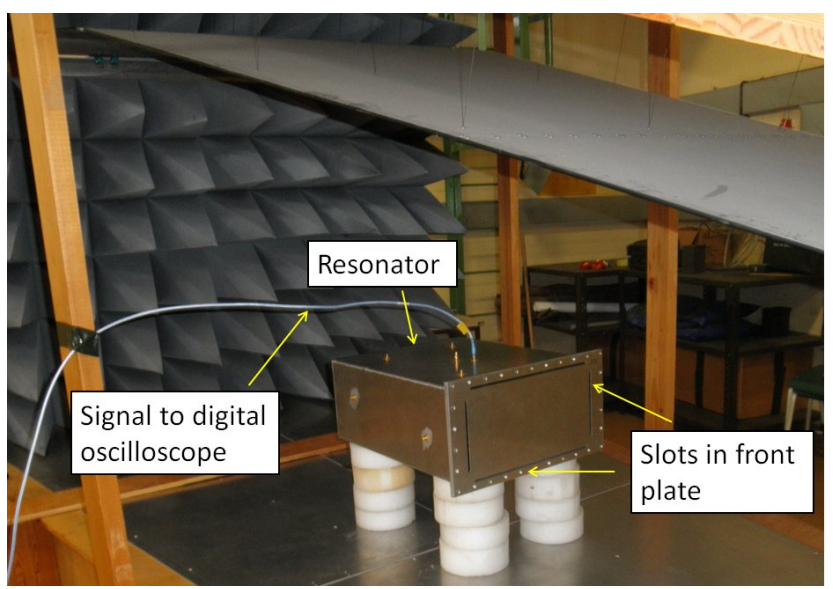

Figure 1. Measurement setup to experimentally observe transient responses to HPEM field excitation: a metallic resonator is placed in an open waveguide which is excited by a transient electromagnetic field. The time domain response of a loop antenna within the resonator is measured via an outside feed by means of a digital oscilloscope.

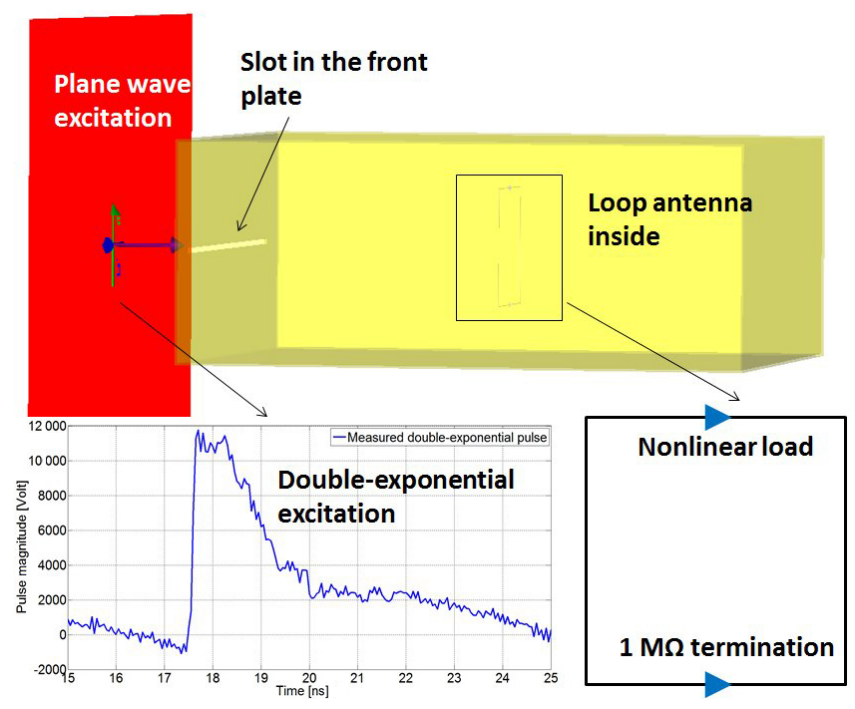

Figure 2. Full-wave model representing the electromagnetic field coupling via a horizontal slot into the resonator if placed into a wave guide. As excitation a plane wave with its time dependency being determined by a measured double-exponential pulse is applied. A quadratic loop antenna of dimensions $10.5 \mathrm{~cm} \times 10.5 \mathrm{~cm}$ is placed inside the resonator and terminated by $1 \mathrm{M} \Omega$ resistor and a nonlinear load.

of the same loop antenna placed inside a resonator is obtained with the help of a numerical full-wave model, as indicated in Fig. 2. These models are completed by the addition of a nonlinear load and allow in Sect. 4 to compare SPICE simulation results with full-wave simulation results. Finally, a short conclusion of the findings is provided by Sect. 5 .

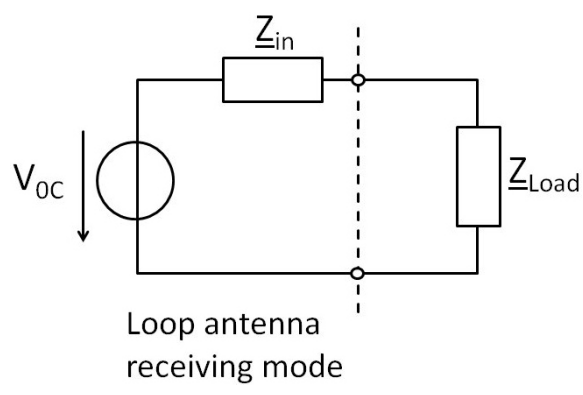

Figure 3. Thevenin equivalent of an antenna in receiving mode, according to Balanis (2005).

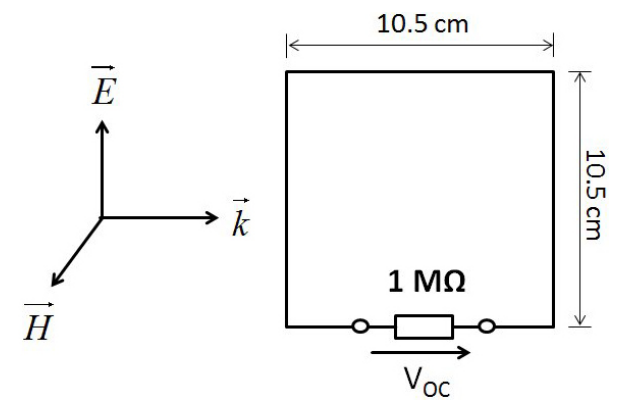

Figure 4. Quantities used for the full-wave modeling of the open circuit voltage in case of the antenna being in receiving mode.

\section{Equivalent circuit model of loop antenna in free space}

In this section an explicit equivalent circuit model of the considered $10.5 \mathrm{~cm} \times 10.5 \mathrm{~cm}$ loop antenna in free space is derived. In order to describe a receiving antenna by an equivalent network it is required to compute the open circuit voltage $V_{\mathrm{oc}}$, which depends from a given excitation, and the antenna input impedance $\underline{Z}_{\text {in }}$, as illustrated in Fig. 3. The open circuit voltage can be obtained from numerical full-wave models where the loop is terminated by a $1 \mathrm{M} \Omega$ resistor as an approximate open port and excited by a known electromagnetic field as indicated in Fig. 4. Then the voltage across the resistor is considered as $V_{\text {oc }}$.

The numerical computation of the input impedance is a standard procedure and, in the present case, has been done using the Method of Moments (MoM), utilizing two different solvers (TET, 2016; Altair, 2016) in frequency domain. The calculated magnitudes of the antenna input impedance are shown in Fig. 5 and the results correlate well in the considered frequency range up to $2 \mathrm{GHz}$. The phase of the input impedance is shown in Fig. 6.

With these values, the following circuit representation is obtained: according to Fig. 5 the first resonance frequency $f_{0}$ of the loop antenna is in the range of $f_{0}=355 \mathrm{MHz}$. Considering the phase at lower frequencies it converges towards $90^{\circ}$ which is characteristic for a parallel RLC-circuit. The resistor $R_{0}$ of this circuit can be derived from Fig. 5, yielding 


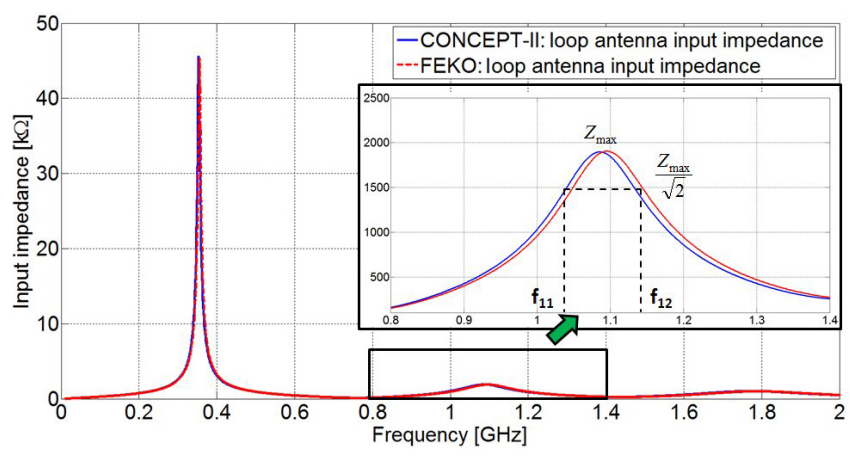

Figure 5. Computed magnitude of the input impedance using two different MoM-solvers (TET, 2016; Altair, 2016).

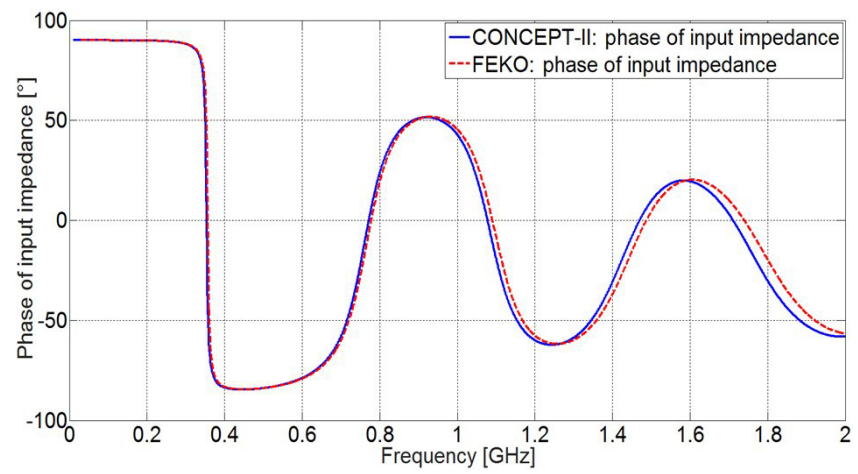

Figure 6. Computed phase of the input impedance using two different MoM-solvers (TET, 2016; Altair, 2016).

approximately $45 \mathrm{k} \Omega$. The corresponding inductance $L_{0}$ and capacitance $C_{0}$ are computed from the input admittance of the parallel resonance circuit,

$Y_{\text {in }}(\omega)=j \omega C_{0}+\frac{1}{j \omega L_{0}}+\frac{1}{R_{0}}$,

where for low frequencies the input admittance depends mainly on the inductance $L_{0}$,

$Y_{\text {in }}(\omega \rightarrow 0) \approx \frac{1}{j \omega L_{0}}$,

and it follows

$\left|Z_{\text {in }}(\omega \rightarrow 0)\right| \approx \omega L_{0}$.

This allows to determine $L_{0}$ from the input impedance at low frequencies. The capacitance $C_{0}$ is obtained from the resonance frequency $f_{0}$ according to

$C_{0}=\frac{1}{4 \pi^{2} f_{0}^{2} L_{0}}$.

In the frequency range up to $2 \mathrm{GHz}$ further resonances occur. In the range of $1.1 \mathrm{GHz}$ the values of the corresponding

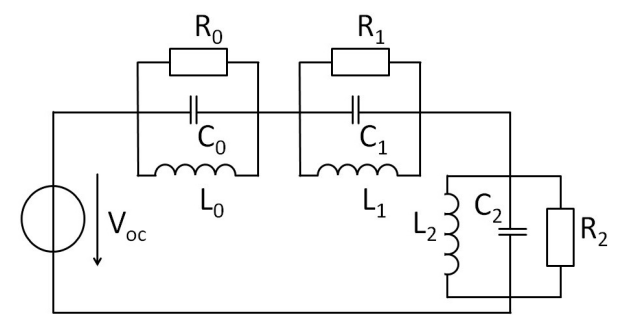

Figure 7. Equivalent circuit of the loop antenna in free space.

Table 1. Computed equivalent circuit element values of the loop antenna in free space.

\begin{tabular}{lrrr}
\hline $\begin{array}{l}\text { Resonance frequency } \\
{[\mathrm{GHz}]}\end{array}$ & $\begin{array}{r}R \\
{[\mathrm{k} \Omega]}\end{array}$ & $\begin{array}{r}L \\
{[\mathrm{nH}]}\end{array}$ & $\begin{array}{r}C \\
{[\mathrm{pF}]}\end{array}$ \\
\hline$f_{0}=0.354$ & 45 & 460 & 0.44 \\
$f_{1}=1.09$ & 1.5 & 30.3 & 0.70 \\
$f_{2}=1.80$ & 1.0 & 12.6 & 0.62 \\
\hline
\end{tabular}

RLC-elements can be derived from relations that involve the resonance bandwidth, compare Fig. 5,

$b_{1}=f_{12}-f_{11}=\frac{1}{2 \pi}\left(\omega_{12}-\omega_{11}\right)=\frac{1}{2 \pi} \frac{1}{R_{1} C_{1}}$.

Analogously, circuit elements related to the third resonance at $1.8 \mathrm{GHz}$ are obtained. The computed RLC-elements are summarized in Table 1 and the corresponding equivalent circuit is depicted in Fig. 7.

If the loop antenna is supplemented by a nonlinear load which is located opposite to the feed then the linear part needs to be considered as an electric dipole antenna, as illustrated in Fig. 8. In contrast to the previous case it is then advisable to derive the equivalent circuit of the electric dipole antenna from its input admittance, see Figs. 9 and 10.

Similar to the previous case, at low frequencies one can determine the capacitor $C_{0}$ from the admittance $Y_{\text {in } 0}$ according to

$\left|Y_{\text {in }}(\omega \rightarrow 0)\right| \approx \omega C_{0}$.

The inductance $L_{0}$ is obtained by a fitting to the resonance frequency $f_{0}=354 \mathrm{MHz}$ that is shown in Fig. 9. From this figure it is also seen that the equivalent circuit model of the dipole antenna should contain, in the considered frequency range, two additional parallel RLC-resonance circuits. The corresponding lumped element values can be computed from the resonance bandwidth again, as indicated in Fig. 9,

$b_{\mathrm{p}}=f_{\mathrm{p} 2}-f_{\mathrm{p} 1}=\frac{1}{2 \pi}\left(\omega_{\mathrm{p} 2}-\omega_{\mathrm{p} 1}\right)=\frac{1}{2 \pi} \frac{1}{\mathrm{RC}}$.

The resulting equivalent circuit lumped-elements are summarized in Table 2. The network representation of the complete nonlinearly loaded loop antenna is shown in Fig. 11. 


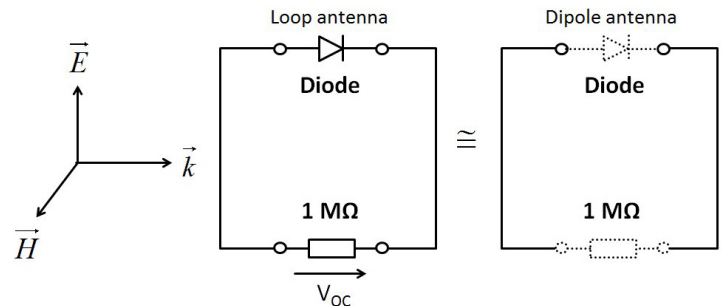

Figure 8. Modeling of the nonlinearly loaded loop antenna in free space. The loop antenna becomes an electric dipole antenna if it is terminated by a nonlinear load opposite to the feed.

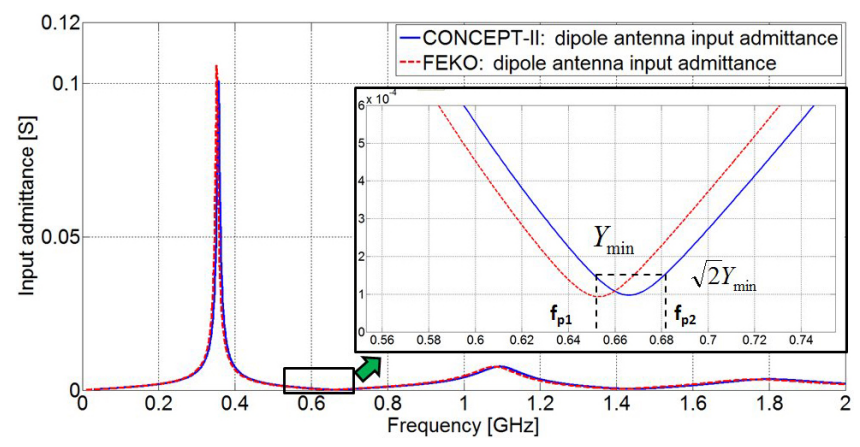

Figure 9. Computed magnitude of the input admittance of the electric dipole antenna using two different MoM-solvers (TET, 2016; Altair, 2016).

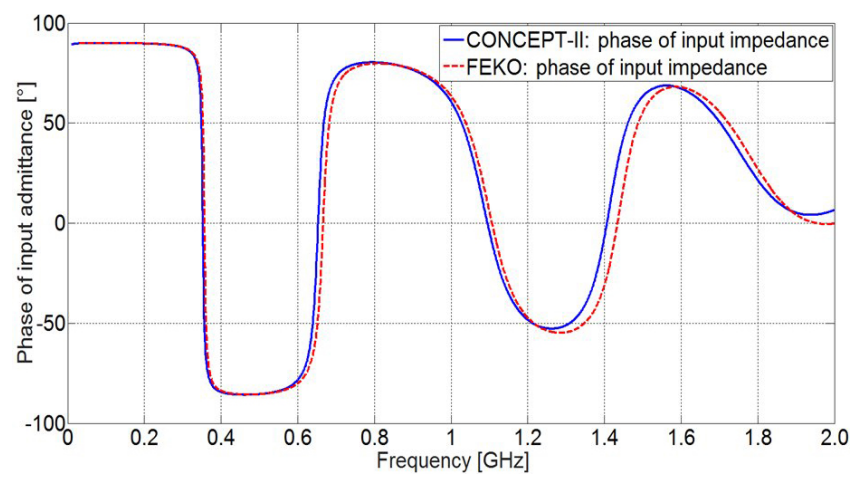

Figure 10. Computed phase of the input admittance of the electric dipole antenna using two different MoM-solvers (TET, 2016; Altair, 2016).

\section{Equivalent circuit model of loop antenna inside a resonator}

In this section modifications to the equivalent circuits are made that are required if the loop antenna is placed inside a resonator. In this case the existence of resonator modes will lead to additional resonances. Using the full-wave solvers based on the MoM (TET, 2016; Altair, 2016) the input impedances of the loop antenna and its electric dipole version, enclosed by a resonator as seen in Fig. 2, have been computed, see Figs. 12 and 14. Due to the interaction be-

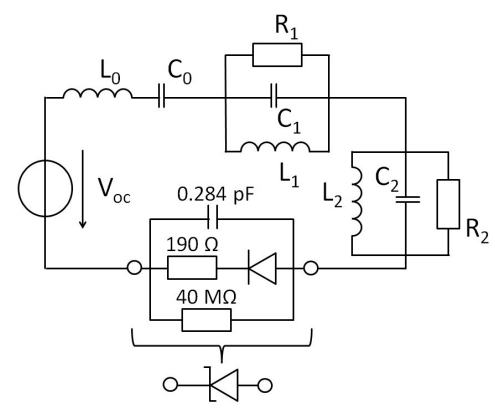

Figure 11. Equivalent circuit of the nonlinearly loaded loop antenna in free space. The representation of the diode follows the model of a realistic diode which is implemented in the full-wave software that will be used in Sect. 4 for time domain computations (CST, 2015).

Table 2. Computed equivalent circuit element values of the electric dipole antenna in free space.

\begin{tabular}{lrrr}
\hline $\begin{array}{l}\text { Resonance frequency } \\
\text { [GHz] }\end{array}$ & $\begin{array}{r}R \\
{[\mathrm{k} \Omega]}\end{array}$ & $\begin{array}{r}L \\
{[\mathrm{nH}]}\end{array}$ & $\begin{array}{r}C \\
{[\mathrm{pF}]}\end{array}$ \\
\hline- & - & 54 & 1.1 \\
$f_{1}=0.637$ & 10.68 & 91 & 0.70 \\
$f_{2}=1.416$ & 2.33 & 17 & 0.74 \\
\hline
\end{tabular}

tween antennas and cavity the low-frequency behavior of the input admittances does change in comparison to the free space situation. This is seen, in particular, for the loop antenna where now it is required to have a series resonance circuit included which is represented by $L_{0}$ and $C_{0}$, see Fig. 13. Also two cavity resonances need to be taken into account. The values of the corresponding lumped elements are summarized in Table 3. Furthermore, the input impedance of the electric dipole shows additional contributions from cavity resonances as well, see Fig. 14. The obtained corresponding values are given in Table 4 . In both cases the serial capacitor $C_{0}$ has been computed from Eq. (6) and the serial inductance $L_{0}$ has been fitted to the numerically calculated input impedance to correctly obtain the first resonance.

\section{Comparison between equivalent circuit models and full-wave models}

It finally is investigated whether full-wave simulations (CST, 2015) of HPEM-induced transient responses at a nonlinear loaded antenna, as depicted in Fig. 2, can be reproduced by the equivalent circuit models developed, if implemented in a SPICE solver (Linear Technology, 2016). It must be noted that the equivalent circuit models of Figs. 11 and 15 require the knowledge of the open-circuit voltage $V_{\text {oc }}$ of the unloaded antenna which is produced by the given excitation (Balanis, 2005). In the present case this open-circuit voltage is obtained from the full-wave solver (CST, 2015) in time 
Table 3. Computed equivalent circuit element values of the loop antenna inside the resonator.

\begin{tabular}{lrrr}
\hline $\begin{array}{l}\text { Resonance frequency } \\
{[\mathrm{GHz}]}\end{array}$ & $\begin{array}{r}L \\
{[\mathrm{k} \Omega]}\end{array}$ & $\begin{array}{r}C \\
{[\mathrm{nH}]}\end{array}$ & $\begin{array}{r}{[\mathrm{pF}]} \\
\hline-\end{array}$ \\
\hline$f_{1}=0.359$ & 215 & 0.75 \\
$f_{2}=0.788$ & 23.65 & 545 & 0.36 \\
$f_{3}=0.812$ & 2.78 & 2.28 & 7.65 \\
$f_{4}=0.951$ & 1.73 & 0.067 & 418 \\
\hline
\end{tabular}

Table 4. Computed equivalent circuit element values of the electric dipole antenna inside the resonator.

\begin{tabular}{lrrr}
\hline $\begin{array}{l}\text { Resonance frequency } \\
{[\mathrm{GHz}]}\end{array}$ & $\begin{array}{r}R \\
{[\mathrm{k} \Omega]}\end{array}$ & $\begin{array}{r}L \\
{[\mathrm{nH}]}\end{array}$ & $\begin{array}{r}C \\
{[\mathrm{pF}]}\end{array}$ \\
\hline- & - & 12 & 1.13 \\
$f_{1}=0.648$ & 630 & 100 & 0.6 \\
$f_{2}=0.800$ & 370 & 6.6 & 6 \\
$f_{3}=0.833$ & 3.344 & 9.6 & 3.8 \\
$f_{4}=0.954$ & 0.510 & 0.66 & 42.1 \\
\hline
\end{tabular}

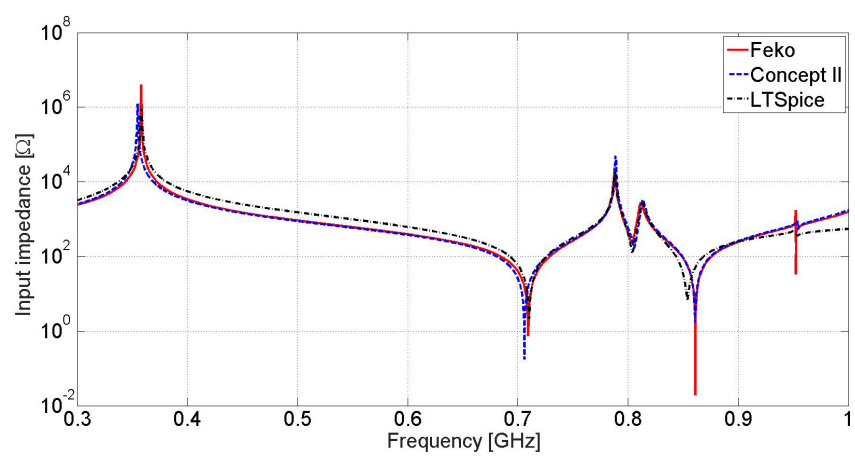

Figure 12. Computed magnitude of the input impedance of the loop antenna placed inside the resonator using two different MoMsolvers (TET, 2016; Altair, 2016). The result obtained from the SPICE model of the calculated equivalent circuit is shown as well.

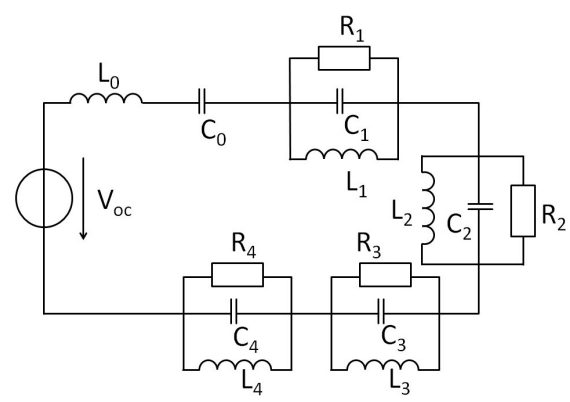

Figure 13. Equivalent circuit of the loop antenna inside the resonator.

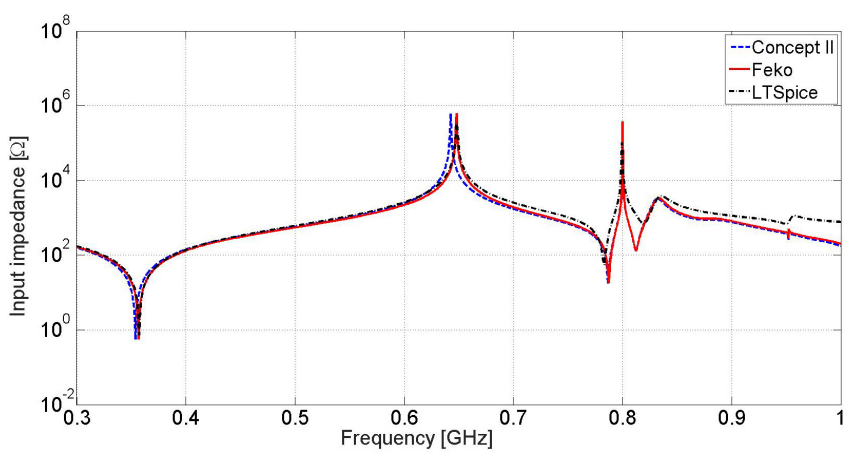

Figure 14. Computed magnitude of the input impedance of the dipole antenna placed inside the resonator using two different MoM-solvers (TET, 2016; Altair, 2016). The result obtained from the SPICE model of the calculated equivalent circuit is shown as well.

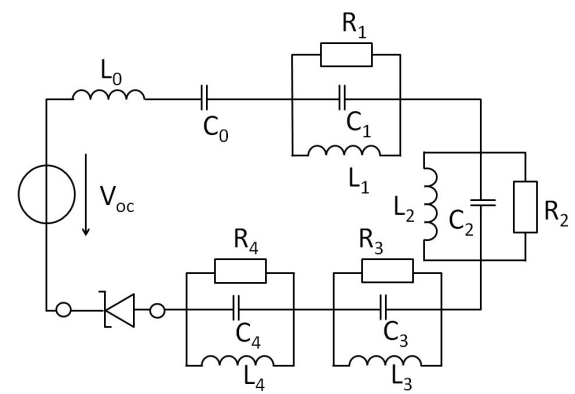

Figure 15. Equivalent circuit of the nonlinearly loaded loop antenna inside the resonator.

domain, that is, the numerically obtained open-circuit voltage can be used as excitation in the SPICE simulation by an import option.

In this way the case of the nonlinearly loaded loop antenna in free space is considered first. In Fig. 16 the comparison of the voltages across the diode computed by the full-wave model and the SPICE model is shown. A nonlinear effect, exhibited by a voltage rectification in time domain, is well captured by both models. This effect has also been observed experimentally (Kotzev et al., 2016). The two solution methods exhibit minor differences in terms of amplitudes and oscillation frequencies while the overall qualitative behavior is reproduced in both cases. In Fig. 17 the voltages across the diode obtained from the full-wave model and the corresponding SPICE model of the loop antenna placed inside the resonator are compared. A quantitative discrepancy between the full-wave and equivalent circuit model computations can be noticed while, again, the rectifying effect is reproduced in both cases. It is not obvious whether the full-wave simulation or the SPICE simulation is closer to physical reality since, from the point of view of a full-wave simulation, the simulation of HPEM-coupling to a nonlinearly loaded antenna within a high-quality resonator is numerically demanding. 


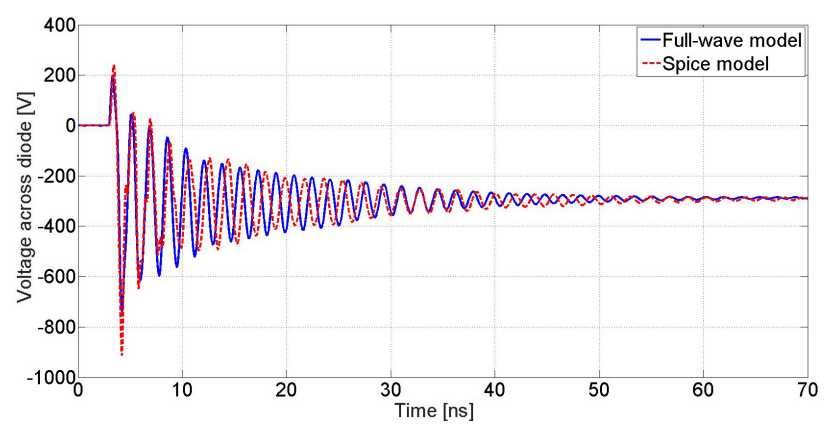

Figure 16. Comparison of the computed voltage across the Schottky diode of the nonlinearly loaded loop antenna in free space. A nonlinear effect expressed by the rectification of the diode's voltage is captured well by both methods used.

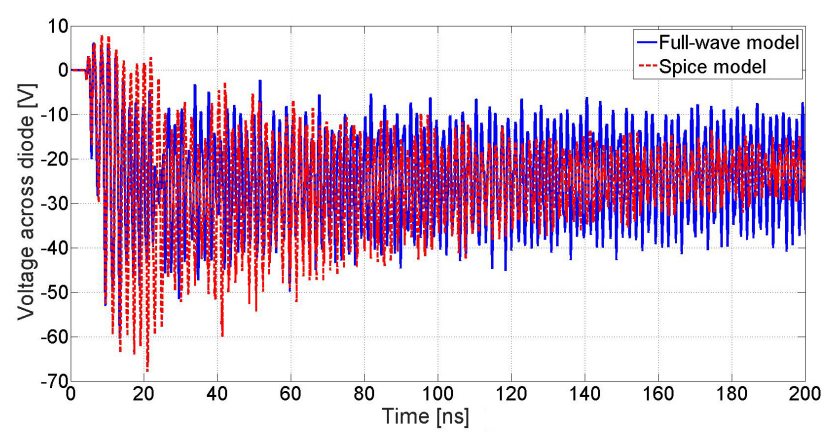

Figure 17. Comparison of the computed voltage across the Schottky diode of the nonlinearly loaded loop antenna placed inside the resonator. The quantitative differences between both methods are larger if compared to the situation in free space.

\section{Conclusions}

In this paper equivalent SPICE models of a nonlinearly loaded loop antenna in free space and inside a metallic resonator have been derived. It was shown that in comparison to full-wave models the equivalent circuit models ensure adequate simulation results and quick computation of the nonlinear effects in the system response induced by transient HPEM-fields.

Data availability. The data presented in this article are available from the authors upon request.

Competing interests. The authors declare that they have no conflict of interest.

Acknowledgements. The authors would like to thank the Electromagnetic Effects and HPEM team at the Bundeswehr Research Institute for Protective Technologies and NBC Protection, Munster, Germany, for their hospitality and reliable support in the EMC laboratory. The research work was funded by the same institute with contract number E/E590/EZ018/CF162.
Edited by: Lars Ole Fichte

Reviewed by: two anonymous referees

\section{References}

Adams, J. J. and Bernhard, J. T.: Broadband equivalent circuit models for antenna impedances and fields using characteristic modes, IEEE T. Antenn. Propag., 61, 3985-3994, https://doi.org/10.1109/TAP.2013.2261852, 2013.

Altair: FEKO Electromagnetic Analysis, Altair, Troy, Michigan, USA, available at: http://www.altairhyperworks.de/, last access: 21 June 2017, 2016.

Balanis, C. A.: Antenna theory: analysis and design, 3rd Edn., John Wiley \& Sons, 2005.

CST: CST EM Studio Version 2013, CST - Computer Simulation Technology AG, Darmstadt, Germany, available at: http://www. cst.com, last access: 21 June 2017, August 2015.

Felsen, L. B., Mongiardo, M., and Russer, P.: Electromagnetic field computation by network methods, Springer Science \& Business Media, https://doi.org/10.1007/978-3-540-93946-7, 2009.

Kotzev, M., Kreitlow, M., and Gronwald, F.: Transient Excitation of Nonlinearly Loaded Resonators and Observation of System Responses in Time Domain, in: 2016 International Symposium on Electromagnetic Compatibility - EMC EUROPE, Wroclaw, Poland, 5-9 September 2016, IEEE, 75-78, https://doi.org/10.1109/EMCEurope.2016.7739287, 2016.

Lee, K. S. H. (Ed.): EMP Interaction: Principles, Techniques, and Reference Data, revised printing, Taylor \& Francis, Washington D.C., 1995.

Linear Technology: LTspice IV, Linear Technology, Milpitas, California, USA, available at: http://www.linear.com/designtools/ software/, last access: 21 June 2017, 2016.

Long, B., Werner, P., and Werner, D.: A simple broadband dipole equivalent circuit model, in: 2000 IEEE Antennas and Propagation Society International Symposium, Salt Lake City, UT, USA, 16-21 July 2000, IEEE, 2, 1046-1049, https://doi.org/10.1109/APS.2000.875401, 2000.

Michalski, K. and Pearson, L.: Equivalent circuit synthesis for a loop antenna based on the singularity expansion method, IEEE T. Antenn. Propag., 32, 433-441, 1984.

Rambabu, K., Ramesh, M., and Kalghatgi, A. T.: Broadband equivalent circuit of a dipole antenna, IEE P. Microw. Anten. P., 146, 391-393, https://doi.org/10.1049/ip-map:19990776, 1999.

Russer, P., Righi, M., Eswarappa, C., and Hoefer, J. W.: Lumped element equivalent circuit parameter extraction of distributed microwave circuits via TLM simulation, in: 1994 IEEE MTT-S International Microwave Symposium Digest, San Diego, CA, USA, USA, 23-27 May 1994, IEEE, 2, 887-890, https://doi.org/10.1109/MWSYM.1994.335215, 1994.

Streable, G. W. and Pearson, L. W.: A numerical study on realizable broad-band and equivalent admittances for dipole and loop antennas, IEEE T. Antenn. Propag., 29, 707-717, 1981.

Tesche, F. M., Ianoz, M. V., and Karlsson, T.: EMC Analysis Methods and Computational Methods, John Wiley \& Sons, New York, 1997.

TET: CONCEPT-II, Institute of Electromagnetic Theory (TET), Hamburg University of Technology (TUHH), available at: http: //www.tet.tuhh.de/concept/uebersicht/, last access: 21 June 2017, 2016. 